\title{
Modelling dynamic bushfire spread: perspectives from the theory of curvature flow
}

\author{
V.-M. Wheeler ${ }^{\text {a }}$, G. E. Wheeler ${ }^{\text {a, }}$,. A. McCoy ${ }^{\mathrm{a}}$ and J. J. Sharples ${ }^{\mathrm{b}}$ \\ ${ }^{a}$ Institute for Mathematics and its Applications, School of Mathematics and Applied Statistics, Faculty of \\ Informatics, University of Wollongong, Wollongong, NSW 2522, Australia
${ }^{\mathrm{b}}$ Applied and Industrial Mathematics Research Group, School of Physical, Environmental and Mathematical \\ Sciences, University of New South Wales Canberra, PO Box 7916, Canberra BC 2610, Australia \\ Email: vwheeler@uow.edu.au
}

\begin{abstract}
It has long been thought that fires are a purely advective phenomenon, but new insights indicate that advection alone is insufficient to adequately account for certain important effects. In this paper we investigate different bushfire scenarios and their relation with newly emerging mathematical results concerning the evolution of curves and surfaces by curvature-dependent speeds. For many fire dynamic effects, even though they arise due to highly complex interactions between fire and atmosphere, it seems that the inclusion of higher-order curvature terms can effectively emulate such behaviours. In previous work, the authors introduced a new cur-vature flow based mathematical model for a fire front, with a perspective toward the modelling of bushfires. The model predicts how the fire front moves by describing the evolution of an isosurface by its mean curvature and a forcing term, consistent with the advective phenomenon. Experimental data and past recorded events of fires have indicated that in certain situations fire fronts with initially finitely many non-smooth points will become smooth. This smoothing effect is captured well by our model. However, in more general settings the smoothness of the evolving isosurface is highly dependent on the smoothness of the initial surface. In real fires the shape and smoothness of the fire front depends on a multitude of factors including the atmospheric conditions, terrain, geographic placement and the type of fuel and its homogeneity. In particular, often the iso-surface of the ignition temperature will not correspond to a smooth surface in the mathematical model. In this paper we consider models that could allow for the propagation of such nonsmooth surfaces. We conclude that a model more complex than our initial mean curvature based model is required for more general applicability. Some analytical results that we have in mind arise from a curvature flow based on a normal evolution with speed given by a homogeneous, symmetric function of the curvatures of the surface. The persistence of non-uniformly convex regions or flat sides, singular parts or ridges of infinite curvature, in the initial surface has been analytically proved and will correspond to some interesting behaviour of nonsmooth initial isosurfaces.
\end{abstract}

Keywords: Curvature flow, partial differential equation, free boundary problem, fire front 


\section{DYNAMIC WILDFIRE PROPAGATION}

A number of wildfires around the world have been shown to exhibit modes of propagation that are manifestly dynamic in nature. The combined effects of wind, terrain and atmospheric conditions have been demonstrated to produce atypical patterns of fire spread (e.g. Sharples et al., 2012). These effects tend to be of great significance to the development of a fire, and in some cases pose a real danger to the community and firefighting crews by their unpredictable nature. Indeed, the dynamic nature of these occurrences means that they are beyond the scope of current operational models that are based on the assumption of quasi-equilibrium fire behaviour. A number of these dynamic effects have been recreated in experiments under controlled conditions.

This paper relates these effects to some of the established behaviour of curves evolving under curvature flows. Although dynamic wildfire propagation undoubtedly arises due to complex three-dimensional interactions between the fire, the terrain and the atmosphere, which are driven by strong pyroconvective effects, it is of interest to represent the effects of such interactions using relatively simple two-dimensional quantities. Indeed, the computational resources required to adequately resolve complex fire-atmosphere interactions over a full three-dimensional domain can be prohibitive in operational contexts. The ability to capture such effects in a meaningful way in a two-dimensional setting, therefore provides for more accurate representation of fire dynamics without the significant computational overheads. Such an approach has been employed to model the dynamic fire spread that arises during the merging of two oblique fire lines (Sharples et al., 2013).

By appealing to the rich literature on curvature flows it is possible to identify behaviours similar to those observed in wildfire situations, and so determine appropriate curvature terms whose addition to the standard model might offer some improvement in their ability to reproduce dynamic effects. We start by reviewing some examples of exotic wildfire behaviour. Our goal is to obtain a better understanding of the types of model that should be considered, depending on the environmental conditions under which a fire is burning.

\subsection{Vorticity-driven lateral spread}

Vorticity-driven lateral spread (VLS), also referred to in the literature as fire channelling, causes a fire to rapidly spread in a direction transverse to the synoptic winds as well as in the usual downwind direction. VLS is characterised by intense lateral and downwind spotting, and production of extensive flaming zones (see Sharples et al. (2010, 2012) and the references therein).

In January 2003 a number of VLS events were observed near Canberra, including those near Pig Hill and Broken Cart shown in Figure 1. These events have been carefully studied (Sharples et al., 2012, 2010) and it has been demonstrated that VLS is caused by a strong wind-terrain-fire interaction (Simpson et al., 2013).

The images in Figure 1 display two distinct fires in which the fire propagated in a direction perpendicular to the wind direction and developed corners and spikes through atypical lateral spread. This behaviour is consistent with two of the analytical properties that have been observed for curvature flows and later discussed in section 2, that is loss of regularity (Subsection 2.2) and loss of convexity (Subsection 2.3).

In Sharples et al. (2010), the authors discuss experiments where similar behaviour is observed. The experiments, performed in the combustion tunnel at the Centro de Estudos Sobre Incendios Florestais, show that an initial weakly convex ignition curve displays, under certain wind and topographical conditions, a
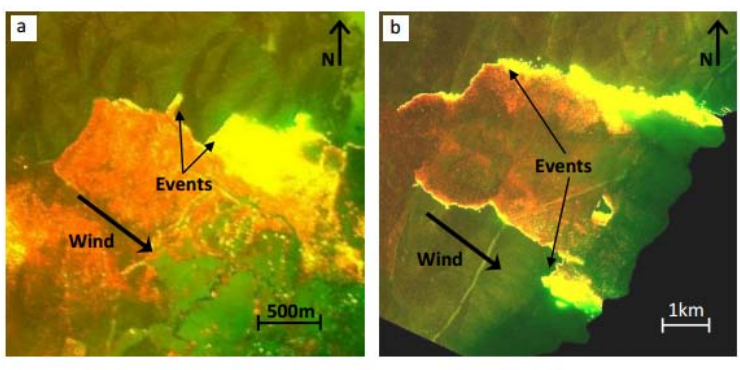

Figure 1. Multispectral line-scan imagery of the Canberra fires 18 January 2003 showing development of corners at: (a) Pig Hill, and (b) Broken Cart.

Source: Sharples et al. (2010). loss of convexity, developing in time as a Yin-Yang curve with lateral spread perpendicular to the wind direction (see Figure 4 in Sharples et al. (2010)). Notable from the same experiments is also the fact that in the absence of wind the fire line preserves convexity as it advances along the slope (see Figure 3 in Sharples et al. (2010) scenario (a)).

\subsection{Development of multiple disjoint parabolic frontal cells from an initial continuous fire front}

Jenkins et al. (2001) presents another unusual behaviour of a fire line that corresponds to phenomena recently studied in curvature flow. For a long enough fire line and certain atmospheric conditions, the fire 
line loses stability and eventually breaks into multiple protrusions or fire heads of parabolic type. This effect is due to the convective updraft column generated by the fire, and is shown schematically in Figure 2.

This sort of behaviour was observed in California in 1985, at the Onion fire in Owens Valley. Jenkins et al. (2001, Figure 7) illustrates the already formed disjoint parabolic frontal cells. Jenkins et al. (2001) noted that once the mean wind died down the fire line reverted back to its initial linear shape.

This behaviour is similar to the loss of convexity and smoothness of an initially convex smooth solution. The return to a regular shape is reminiscent of fourth and higher-order curvature flow (see Blatt (2010)).

\section{RESULTS FROM THE THEORY OF CURVATURE FLOW}

\subsection{Context in fire front modelling}

In earlier models of fire fronts as evolving curves in the plane, we included a small curvature term in the front velocity in order to account for various ob-

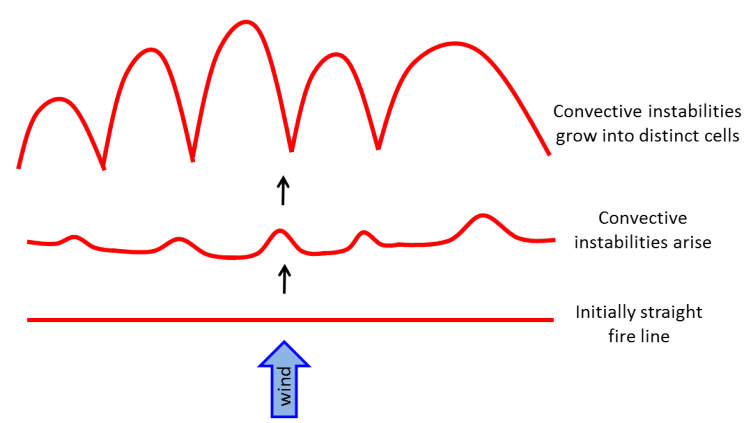

Figure 2. Schematic illustrating loss of fire line convexity, such as was observed in the 1985 Onion sage brush fire (Jenkins et al. (2001)). served physical phenomena, such as jump velocity in merging fire fronts and the instantaneous smoothing of corner-fires (Sharples et al., 2013). A higher-dimensional model was also proposed by Wheeler et al. (2013) where dependence on the mean curvature of the evolving front was included in its velocity.

In this paper we consider examples in dimension $n=2$, that is moving surfaces and $n=3$, corresponding to moving hypersurfaces. For the surfaces case, the object evolving with speed depending on its curvature is a smooth model of the isosurface obtained by mapping the burning point of the fuel. Surface examples pertaining to loss of regularity and persistence of flat sides are considered in section 2.3 and 2.5. For hypersufaces, the evolving object will be the area enclosed by the fire. Examples corresponding to this higher dimensional case are found in section 2.4, which discusses loss of convexity. The construction of surface examples for loss of convexity is still open. The higher dimensional problems are able to include into the model the height of a fire front that moves into a 3 -dimensional fuel space.

There is an enormous variety of possibilities; many more variations on curvature exist instead of simply the mean curvature of a surface, or the standard curvature of a plane curve. The underlying idea is to use insights from the theory of curvature flow of hypersurfaces to devise new possibilities for this curvature term. For example, some observed fire fronts exhibit loss of regularity in certain conditions, and so Theorem 1 (presented below) gives us a number of sufficient conditions on the curvature term in the flow that yields this behaviour. It is important to note that this does not include the mean curvature, and so these are new models.

Consider the behaviour of solutions $X: M^{n} \times[0, T) \rightarrow \mathbb{R}^{n+1}$ ( $n=2$ or 3 ) of curvature flow equations of the form

$$
\frac{\partial X}{\partial t}(x, t)=-F(\mathcal{W}(x, t)) \nu(x, t)
$$

where $X$ is a smooth family of isometric immersions (that is, $X$ is smooth and for each $t$ the map $X_{t}=$ $X(\cdot, t)$ is an isometric immersion), $M^{n}$ is a smooth manifold, $\nu(x, t)$ is the outer unit normal to the image hypersurface $M_{t}=X_{t}\left(M^{n}, t\right)$ at $X(x, t)$ and $\mathcal{W}(x, t)$ is the Weingarten map of $M_{t}$, that is the differential of the choice of unit normal vector field on the hypersurface. This linear map contains the curvature information for a hypersurface and can be expressed in matrix form by computing the normal projections of the second derivatives of the parametrisation $X$. The eigenvalues of its matrix form are the principal curvatures of $M_{t}$. The sum of the principal curvatures squared is called the norm of the second fundamental form squared and it is denoted by $|A|^{2}$.

In most physically relevant flows, the function $F$ is geometrically invariant, so $F(\mathcal{W})=f\left(\kappa_{1}, \kappa_{2}, \kappa_{3}\right)$, for some symmetric function $f$ of the principal curvatures $\kappa_{1}, \kappa_{2}$ and $\kappa_{3}$. In view of symmetry, we write $f(\kappa)$ where $\kappa=\left\{\kappa_{1}, \kappa_{2}, \kappa_{3}\right\}$ is the set of principal curvatures of $M_{t}$ at each point $(x, t)$. Relationships between derivatives of $F$ and of $f$ are reasonably well known (see for example Glaeser (1963); Schwarz (1975)). 
We further restrict the class of allowable speeds $F$ by requiring $F$ to be smooth and homogeneous of some strictly positive degree $\alpha$ : for any $k>0$,

$$
f(k \kappa)=k^{\alpha} f(\kappa) .
$$

This amounts to the requirement that the flow (1) has an invariance under spatial scalings, which is a natural assumption for physical phenomena such as fire fronts.

Other conditions that are typically imposed on $f$ include:

(i) $f$ is smooth, symmetric and defined at least on the positive cone $\Gamma_{+}=\left\{\kappa \in \mathbb{R}^{n}: \kappa_{i}>0\right.$ for all $i=$ $1,3\}$.

(ii) $f$ is strictly increasing in each argument: $\dot{f}^{i}:=\frac{\partial f}{\partial \kappa_{i}}>0$ for each $i=1,3$, at every point of its domain. Amongst other reasons, this is needed for short-time existence of a solution to (1).

(iii) $f$ is strictly positive on $\Gamma_{+}$and normalised; $f(1,1,1)=1$.

\section{Remarks.}

1. Strict positivity follows from the first three conditions and so (iii) is indeed merely a normalisation.

2. In recent work (Andrews et al., 2014) condition (ii) has been weakened to a condition that $f$ is increasing but not necessarily differentiable.

3. We would really like to incorporate a lower order term into (1) which would dominate the speed in regions of the front where the curvature is small. It remains to check that the behaviour for flows of the form (1) that we outline below carries over to flows with such an additional term. More generally, flows with arbitrary lower order terms have been considered before in the setting of convex initial hypersurfaces (see for example Li and Salavessa (2008); Li et al. (2009); Wu et al. (2010)), although to the best of our knowledge properties like those we explore below have not been investigated.

\subsection{Examples}

In order to indicate the variety still allowed by the above conditions, we give some typical families of flow speeds that satisfy all of the given requirements. Most results are available when the speed is homogeneous of degree 1.

Elementary symmetric functions. For evolving surfaces these are $H$ (the mean curvature) and $K$ (the Gauss curvature).

Rational combinations of elementary symmetric functions. For surfaces this includes $\frac{K}{H}, \frac{H^{3}}{K}$ provided the setting is such that the conditions including positivity are maintained under the flow.

Power means.

$$
f=H_{r}=\left(\frac{1}{n} \sum_{i=1}^{n} \kappa_{i}^{r}\right)^{\frac{1}{r}}
$$

for any real $r$. Special cases include $r=1\left(H_{1}=H\right.$, the mean curvature), $r=2\left(H_{2}=\sqrt{\kappa_{1}^{2}+\kappa_{2}^{2}+\kappa_{3}^{2}}=\right.$ $|A|$, the so-called norm of the second fundamental form), $r=-1\left(H_{-1}=\left(\kappa_{1}^{-1}+\kappa_{2}^{-1}+\kappa_{3}^{-1}\right)=\frac{K}{H}\right.$, the harmonic mean curvature).

Convex and weighted geometric combinations. Any convex combination of the above examples is again allowable. Weighted geometric means, that is

$$
f=\prod_{i=1}^{k} f_{i}^{\alpha_{i}}, \text { where } \alpha_{i}>0 \text { and } \sum_{i=1}^{k} \alpha_{i}=1 \text { and } f_{1}, \ldots, f_{k} \text { come from the above examples. }
$$

Note that all of these except for the power means with $r>1$ are concave, and all except for the power means with $r<-1$ are inverse-concave. We call an $f$ inverse-concave if the associated function $f_{*}$ defined by

$$
f_{*}\left(r_{1}, r_{2}, r_{3}\right)=f\left(\kappa_{1}^{-1}, \kappa_{2}^{-1}, \kappa_{3}^{-1}\right)^{-1}
$$

is concave on its domain $\Gamma_{+}$. Here $r_{i}$ are the principal radii of curvature, $r_{i}=\frac{1}{\kappa_{i}}$, that are well-defined for convex surfaces. Behaviour of the $f_{*}$ function is critical in determining whether a curvature flow of positive homogeneity preserves flat sides, as we describe later. 


\subsection{Loss of regularity}

The mean curvature flow and many other second-order, fully nonlinear curvature flows, shrink and smooth uniformly convex hypersurfaces to points (see for example Huisken (1984); Tso (1985); Andrews (1994); Andrews et al. (2013)). However, contrary to expectation, the class of curvature flows that are able to drive a smooth, uniformly convex hypersurface to a curvature singularity before shrinking to a point is also quite large, including many natural examples. Since we are interested here in evolving surfaces, example speeds that can evolve convex surfaces to curvature singularities include powers $\alpha \neq 1$ of the harmonic mean curvature (Andrews et al., 2013, Corollary 1). This is particularly surprising since when $\alpha=1$ such flows drive convex surfaces to round points Andrews (1994).

The technical theorem below gives sufficient conditions for which an initial smooth front will develop corners, that is lose the smoothness property before extinction:

Theorem 1 (Andrews et al. (2013)). Let $\alpha \neq 1$. Suppose that

$$
\dot{f}_{*}^{i}(\tau)>0 \text { if } \tau \in \partial \Gamma_{+} \text {with } f_{*}(\tau)>0
$$

for each $i$ and $f_{*}$ is smooth near $\tau$; and

$$
\lim _{\tau \rightarrow \tau_{+} \in \partial \Gamma_{+}} f_{*}(\tau) \neq 0
$$

for all $\tau_{+} \in \partial \Gamma_{+}$. Then there exist smooth, uniformly convex initial hypersurfaces for which the solution of the flow (1) has $\max _{M_{t}}|A|^{2} \rightarrow \infty$ as $t \rightarrow T$, but the inradius of $M_{t}$ does not approach zero as $t \rightarrow T$.

The nonvanishing inradius (the radius of the largest Euclidean ball that can be fitted in the interior of the hypersurface without intersecting it) tells us that the loss of smoothness does not occur due to the enclosed fuel being consumed.

Front velocity for loss of regularity and smoothing effect. By replacing the mean curvature term from Wheeler et al. (2013) with a positive power of the harmonic mean curvature, we are able to exhibit a flow which has both instantaneous smoothing and loss of regularity. If $\alpha>0, \alpha \neq 1$ and $\epsilon>0$, then the flow has velocity:

$$
V=\left[-\varepsilon\left(\frac{1}{\sum_{i=1}^{n} \kappa_{i}^{-1}}\right)^{\alpha}+1\right] \nu,
$$

If we require the degree of homogeneity to be one, then there are still examples, as the following theorem shows.

Theorem 2 (Andrews et al. (2013)). Let $\alpha=1$. Suppose that (5) holds and

$$
\left.f_{*}\right|_{\partial \Gamma_{+}} \text {is not inverse-concave. }
$$

Then there exist smooth, uniformly convex initial hypersurfaces for which the solution of the flow (1) has $\max _{M_{t}}|A|^{2} \rightarrow \infty$ as $t \rightarrow T$, but the inradius of $M_{t}$ does not approach zero as $t \rightarrow T$.

\subsection{Loss of convexity}

Loss of convexity has been observed in fire front behaviour (Jenkins et al., 2001; Sharples et al., 2012). For the second-order curvature flows in Andrews et al. (2013), the examples of loss of convexity apply only for hypersurfaces of dimension at least 3 , although it is still possible that some second-order flows of surfaces could lead to loss of convexity. Moreover, flows containing a global term can have different behaviour (see, for example Cabezas-Rivas and Miquel (2014)). On the other hand, it is typical that convexity is lost for higher-order curvature flow (Blatt, 2010).

When inverse-concavity fails, uniform convexity of the solution can be lost under the flow (1). An example of a flow speed for which this occurs is

$$
f(x, y, z)=\frac{x y}{\sqrt{x^{2}+y^{2}}}+\frac{x z}{\sqrt{x^{2}+z^{2}}}+\frac{y z}{\sqrt{y^{2}+z^{2}}} .
$$

The precise statement gives the existence of an initial uniformly convex hypersurface for which convexity is lost under the flow, since the smallest principal curvature becomes negative and remains so. 
V.-M. Wheeler, et al., Modelling dynamic bushfire spread: perspectives from the theory of curvature flow

Theorem 3 (Andrews et al. (2013)). Suppose that

$$
\dot{f}^{i}(\kappa)>0 \text { if } \kappa \in \partial \Gamma_{+} \text {with } f(\kappa)>0
$$

for each $i$ and $f$ is smooth near $\kappa$; and

$$
\left.f\right|_{\partial \Gamma_{+}} \text {is not inverse-concave. }
$$

Then there exist smooth, uniformly convex initial hypersurfaces for which the solution of the flow (1) has negative smallest principal curvature for all sufficiently small $t>0$.

\subsection{Persistence of flat sides}

Persistence or instantaneous movement of flat sides is another property of curvature flow that distinguishes certain classes of flow speeds. We may think of a flat side as an open, connected, non-empty, two-dimensional set of points in $M_{0}$ where both principal curvatures are everywhere equal to zero. As with loss of regularity, the case of degree-one homogeneity is critical. We begin with a result for homogeneity $\alpha \in(0,1)$.

Theorem 4 (Andrews et al. (2013)). Let $0<\alpha<1$. Suppose $M_{0}$ is smooth and weakly convex with a flat side. Under (1), the flat side immediately moves and for all $t>0$ sufficiently small $M_{t}$ is convex.

As flat sides of fire fronts often vanish, we are mostly interested here in curvature flows for which flat sides immediately disappear. In view of this result, it appears that curvature contraction flows with speeds homogeneous of degree $\alpha<1$ are likely to be most relevant to fire front modelling.

In stark contrast to this is the case of $\alpha>1$, where flat sides always persist.

Theorem 5 (Andrews et al. (2013)). Let $\alpha>1$. Suppose $M_{0}$ has a flat side; that is, an open connected non-empty set of points in which the curvature vanishes. Then the flat side persists at least for an amount of time proportional to an absolute constant depending only on homogeneity multiplied by $d^{1+\alpha}$, where $d$ is the diameter of the largest disc contained in the flat side.

The critical case allows the following simple dichotomy.

Theorem 6 (Andrews et al. (2013)). Let $\alpha=1$. Flat sides move under (1) if and only if $f_{*}=0$ on $\partial \Gamma_{+}$.

For fire fronts, we expect flat sides to move, implying they become convex, and therefore suitable speeds should satisfy $f_{*}=0$ on $\partial \Gamma_{+}$. Equivalently, $f$ should satisfy $\lim _{x \rightarrow \infty} f(x, 1)=\infty$. Some examples of such speeds for surfaces are

- $F=H$, so $f(\kappa)=\kappa_{1}+\kappa_{2}$ and $\lim _{x \rightarrow \infty} f(x, 1)=\lim _{x \rightarrow \infty} x+1=\infty$;

- as a generalisation, $F=H_{r}$ for any $r>0$, then $\lim _{x \rightarrow \infty} f(x, 1)=\left[\frac{1}{2}\left(x^{r}+1\right)\right]^{\frac{1}{r}}=\infty$;

- $F=\sqrt{K}$, so $f(\kappa)=\sqrt{\kappa_{1} \kappa_{2}}$ and $\lim _{x \rightarrow \infty} f(x, 1)=\lim _{x \rightarrow \infty} \sqrt{x}=\infty$.

By contrast, the other seemingly natural degree-one homogeneous speed $F=\frac{K}{H}$ has $f(x, 1)=\frac{x}{1+x}$ that approaches 1 as $x \rightarrow \infty$, so flat sides can remain flat.

\section{CONCLUSIONS}

This paper discussed a number of instances of dynamic wildfire propagation, highlighting the associated exotic behaviour of the evolving perimeter, and considering such from the perspective of some recent advances in the theory of curvature flows. In particular, a number of instances of wildfire behaviour that are in close analogy with characteristics of nonlinear curvature flows, such as loss of smoothness and convexity, were discussed. Such behaviours have only recently been rigorously investigated in the mathematical literature. After reviewing relevant parts of these developments modified models, which are known to exhibit the observed phenomena, were proposed for study. There are significant technical limitations on the theory currently available; for example, requiring degree-one homogeneity in the speed. There are two clear directions for future work: (1) rigorous comparison of numerical simulation of the flows proposed with experimental and wildfire data; and (2) weakening of requirements (such as positive homogeneity of the speed) that will allow investigations into flows that, for example, are of expansion type, or that include lower order terms. 
V.-M. Wheeler, et al., Modelling dynamic bushfire spread: perspectives from the theory of curvature flow

\section{REFERENCES}

Andrews, B., A. Holder, J. McCoy, G. Wheeler, V.-M. Wheeler, and G. Williams (2014). Curvature contraction of convex hypersurfaces by nonsmooth speeds. Journal für die reine und angewandte Mathematik (Crelles Journal). Online First.

Andrews, B. H. (1994). Contraction of convex hypersurfaces in euclidean space. Calc. Var. Partial Differential Equations 2(2), 151-171.

Andrews, B. H., J. A. McCoy, and Y. Zheng (2013). Contracting convex hypersurfaces by curvature. Calc. Var. 47, 611-665.

Blatt, S. (2010). Loss of convexity and embeddedness for geometric evolution equations of higher order. Journal of Evolution Equations 10(1), 21-27.

Cabezas-Rivas, E. and V. Miquel (2014). Non-preserved curvature conditions under constrained mean curvature flows. arXiv preprint arXiv:1412.8143.

Glaeser, G. (1963). Fonctions composées différentiables. Ann. of Math. (2) 77, 193-209.

Huisken, G. (1984). Flow by mean curvature of convex surfaces into spheres. J. Differential Geom. 20(1), 237-266.

Jenkins, M. A., T. Clark, and J. Coen (2001). Coupling atmospheric and fire models. In E. A. Johnson and K. Miyanishi (Eds.), Forest fires: behavior and ecological effects. Academic Press: San Diego, CA, USA.

Li, G. and I. Salavessa (2008). Forced convex mean curvature flow in euclidean spaces. Manuscripta Math. 126(3), 333-351.

Li, G., L. Yu, and C. Wu (2009). Curvature flow with a general forcing term in euclidean spaces. J. Math. Anal. Appl. 353(3), 508-520.

Schwarz, G. W. (1975). Smooth functions invariant under the action of a compact lie group. Topology 14, 63-68.

Sharples, J. J., R. H. McRae, and S. R. Wilkes (2012). Wind-terrain effects on the propagation of wildfires in rugged terrain: fire channelling. International Journal of Wildland Fire 21(3), 282-296.

Sharples, J. J., I. N. Towers, G. E. Wheeler, V.-M. Wheeler, and J. A. McCoy (2013). Modelling fire line merging using plane curvature flow. In J. Piantadosi, R. S. Anderssen, and J. Boland (Eds.), 20th International Congress on Modelling and Simulation, pp. 256-262. Modelling and Simulation Society of Australia and New Zealand.

Sharples, J. J., D. X. Viegas, C. G. Rossa, and R. H. McRae (2010). Small-scale observations of atypical fire spread caused by the interaction of wind, terrain and fire. In Proceedings of the VI International Conference on Forest Fire Research, pp. 15-18.

Simpson, C. C., J. J. Sharples, J. P. Evans, and M. F. McCabe (2013). Large eddy simulation of atypical wildland fire spread on leeward slopes. International Journal of Wildland Fire 22, 282-296.

Tso, K.-S. (1985). Deforming a hypersurface by its gauss-kronecker curvature. Comm. Pure Appl. Math. 38, $867-882$.

Wheeler, V.-M., J. A. McCoy, G. E. Wheeler, and J. J. Sharples (2013). Curvature flows and barriers in fire front modelling. In J. Piantadosi, R. S. Anderssen, and J. Boland (Eds.), 20th International Congress on Modelling and Simulation, pp. 297-303. Modelling and Simulation Society of Australia and New Zealand.

Wu, C., D. Tian, and G. Li (2010). Forced flow by powers of the $m$ th mean curvature. Arm. J. Math. 3(2), 61-92. 